Communications in Physics, Vol. 14, No. 2 (2004), pp. 111-118

\title{
CONTINUOUSLY TUNING DISTRIBUTED BRAGG REFLECTOR ERBIUM DOPED FIBER LASER
}

\author{
TRAN THI TAM, VU NGOC CHAM \\ Faculty of Technology, Hanoi National University \\ DANG QUOC TRUNG, TRAN ANH VU, AND LE HUU MINH \\ Lab. of Fiber laser, Institute of Materials Science, VAST
}

\begin{abstract}
The linear configuration single-mode Distributed Bragg Reflection (DBR) Erbium doped Fiber laser has been tested. The cavity length of the laser utilizing intracore Bragg reflectors, no longer than one centimetre and the grating bandwidth is below $\sim 0.2$ $\mathrm{nm}$. The 1.55- $\mu \mathrm{m}$ emission wavelength of the laser can be continously tuned within $2 \mathrm{~nm}$.
\end{abstract}

\section{INTRODUCTION}

The Erbium doped fiber (EDF) lasers have been a subject of intensive research because of their potential application in various fields such as communication, spectroscopy, sensing and medicine [1]. In particular, single longitudinal mode, narrow linewidth laser radiation in $1,55-\mu \mathrm{m}$ region with the capability of being continuously tuned its wavelength without longitudinal mode hopping has attracted special interest owing to its application in telecommunication, wavelength-division multiplexing and high resolution spectroscopy. Several different configurations of single mode, narrow linewidth EDF lasers have been constructed, including ring resonators [2], Fox-Smith resonators [3], coupled-linear cavity resonators [4], or intra-core Bragg reflectors [5]. The interest in the linear resonator configuration belongs primarily to engineering simplicity, while maintaining high performance and efficiency, increased reliability, and decreased cost. In this paper we report upon the research of linear cavity, single longitudinal mode EDF laser using intra-core Bragg reflectors for cavity feedback and mode discrimination. Such configuration has an ability to tune the laser operating wavelength continuously without longitudinal mode hopping if both the gratings and intervening fiber are uniformly stretched or heated .

Since the single-mode fiber laser only lases in the lowest-loss mode, the stability of the single-mode solution is important as the fiber optical path is strongly affected by thermal, acoustic, and strain variation perturbations making the cavity length difficult to fix, and small length variation $\lambda / 2 n$ will shift the resonance one free-spectral-range.

Given the gain and loss mechanisms within the resonator, the constraints required to achieve single-mode operation within a linear-cavity fiber laser with intra-core Bragg reflectors can be found.

\section{THEORETICAL ANALYSIS}

\section{Bragg Gratings Characteristics}

The reflectivity of rectangular Bragg gratings that are holographically written in a single-mode fiber has been modeled using coupled mode theory, as function of wavelength 
and grating length, is given by [6]

$$
R\left(L_{g}, \lambda\right)= \begin{cases}\frac{\Omega^{2} \sinh ^{2}\left(S L_{g}\right)}{\Delta \beta^{2} \sinh ^{2}\left(S L_{g}\right)+S^{2} \cosh ^{2}\left(S L_{g}\right)} & \text { for } \Omega^{2} \succ \Delta \beta^{2} \\ \frac{\Omega^{2} \sin ^{2}\left(Q L_{g}\right)}{\Delta \beta^{2}-\Omega^{2} \cos ^{2}\left(Q L_{g}\right)} & \text { for } \Omega^{2} \prec \Delta \beta^{2}\end{cases}
$$

where $\lambda$ - wavelength, $L_{g}$ - grating length, $\Omega$ - coupling coefficient, $\Delta \beta=\beta-(\pi / \Lambda)$, $\beta=\frac{2 \pi}{\lambda} n$ is the eigen-propagation constant, $\Lambda$ - grating period, $S=\left(\Omega^{2}-\Delta \beta^{2}\right)^{1 / 2}$, and $Q=\left(\Delta \beta^{2}-\Omega^{2}\right)^{1 / 2}$. The equation is valid with the assumption of weak guiding, negligible absorption and negligible coupling of propogation modes to radiation modes. The coupling coefficient $\Omega$ is dependent on the induced index perturbation, which is a function of fiber composition and grating exposure.

Gratings reflectivity ranging between $0-100 \%$, and minimum bandwidths ranging within the 0.1- $0.2 \mathrm{~nm}$ region may be fabricated in erbium doped germanosilicate fiber. In this case, the refractive index's change $\Delta n$ as results of UV light exposure is generally in the order of $\approx 5 \times 10^{-4}$.

The fiber laser of a resonator length $L_{c}$ will lase at wavelengths that meet the cavity resonance condition:

$$
\lambda_{0}=\frac{2}{m} n L_{C}
$$

where $\lambda_{0}$ is a resonance wavelength, $n$ is the refractive index, and $m$ is an integer.

After the Bragg condition of a grating reflector, the relation between wavelength of the reflected radiation line center $\lambda_{B}$ and the grating period $\Lambda$ is following:

$$
\lambda_{B}=2 n \Lambda
$$

As known the condition for single-mode operation is that i) the dominant lasing mode is at center of grating region, in which the gain difference is negligible, ii) the next mode must be de-tuned out off the grating region. Therefore the free spectral range $\Delta \beta_{F S R}$ should be bigger than half of the grating region:

$$
\Delta \beta_{F S R}=\frac{\pi}{L_{c}}
$$

For the Bragg gratings' width of about $0.2 \mathrm{~nm}$, the laser cavity length is estimated about $8 \mathrm{~mm}$.

\section{Single - mode linear cavity EDF laser design}

To get single - mode linear cavity EDF Laser, we will follow the basic mode selection principle by applying mode selecting elements (gratings). When narrow-band Bragg reflectors are used for cavity feedback, by choosing adequate fiber length to get appropriate free spectral range (FSR) we can realize single longitudinal mode operation. Fig. 1 shows energy diagram for $\mathrm{Er}^{3+}$-ions in silica. The laser transition is between two states ${ }^{4} \mathrm{I}_{13 / 2}$ and ${ }^{4} \mathrm{I}_{15 / 2}$. When pump the ED fiber with the $980 \mathrm{~nm}$ photons the excited state absorption may be ignored. The three-level lasing process is: the ground-state ions that 
absorb photons are transferred to ${ }^{4} \mathrm{I}_{11 / 2}$ level, then rapidly decay to upper laser ${ }^{4} \mathrm{I}_{13 / 2}$ level. From ${ }^{4} \mathrm{I}_{13 / 2}$ level the ions return to ground level ${ }^{4} \mathrm{I}_{15 / 2}$ through spontaneous and/or stimulated emission. Part of ions in ground state may be exited to ${ }^{4} \mathrm{I}_{13 / 2}$ level by reabsorbing the emitted signal photons. The medium gain may be estimated based on rate equation for three level systems. Followed B.E.A. Saleh [7], we have the gain for steady state:

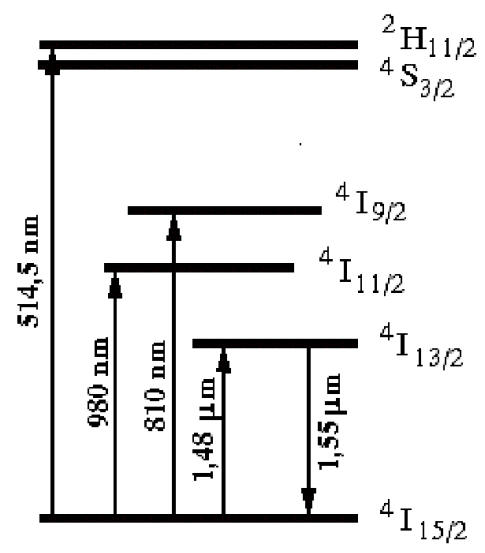

Fig. 1. The energy diagram for $\mathrm{Er}^{3+}$-ions in silica

$$
\alpha=\frac{\alpha_{0}}{1+\frac{\sum_{k} I_{k}}{I_{\text {sat }}}}
$$

where

$$
\alpha_{0}=N_{0} \frac{\sigma_{e} \tau_{2} W_{p}-\sigma_{a}}{1+\tau_{2} W_{p}}
$$

and

$$
I_{s a t}=\frac{h v_{s}\left[W_{p}+\frac{1}{\tau_{2}}\right]}{\left(\sigma_{a}-\sigma_{e}\right)}
$$

$W_{p}=\sigma_{p} I_{p} / h v_{p}, \quad W_{a}=\sigma_{a} I_{s} / h v_{s}, \quad W_{e}=\sigma_{e} I_{s} / h v_{s}, N_{0}$ is total ions population density, $\sigma_{p}, \sigma_{a}, \sigma_{e}$ are pump absorption, signal absorption and signal emission cross sections, respectively; $I_{p}, I_{s}$ - pump and signal intensities; $\tau_{2}$ - upper level's life time; $k$ is the index of the lasing mode, and $I_{k}(x)$ is the modal intensity in the fiber.

The transient energy density per mode within the resonator can be written as

$$
\frac{d E_{k}(x)}{d t}=\alpha I_{k}(x)-\gamma_{k} I_{k}(x)
$$

where $\alpha$ is the modal gain, $\gamma_{k}$ is the modal loss. For fiber lasers in which both fiber propagation and grating losses are negligible (which is the case of intra-core Bragg gratings), $\gamma$ is given by

$$
\gamma=-\ln \left[R_{1}(\lambda) R_{2}(\lambda)\right]
$$

where $R_{1}(\lambda)$ and $R_{2}(\lambda)$ are the reflectivity spectra of the Bragg gratings.

To get stable single-mode operation, we should consider the condition for the potential lasing of two modes, since the stable single-mode operation is dependent on both the gain and the loss for the two lowest loss cavity modes. The gain of the erbium-doped fiber is much broader than grating bandwidth, so we can consider it to be homogeneous and constant over the narrow grating's bandwidth, and the gain is assumed to be identical for 
both modes. Mode discrimination must therefore result from the cavity loss mechanism. When narrow-band intra-core Bragg reflectors are used for cavity feedback, the differential loss as a function of wavelength is naturally imposed on the cavity. Two neighboring longitudinal modes will therefore see different cavity losses. The gain for two modes may be rewritten as:

$$
\alpha=\frac{\alpha_{0}}{1+\frac{I_{1}(x)+I_{2}(x)}{I_{\text {sat }}}} \approx \alpha_{0}\left[1-\frac{I_{1}(x)}{I_{\text {sat }}}-\frac{I_{2}(x)}{I_{\text {sat }}}\right]
$$

Substituting (10) into (8) and making integration over the length of the fiber gives the intensity of each mode at steady state [5]:

$$
I_{1,2}=\frac{I_{\text {sat }}}{3}\left(1-\frac{\gamma_{1,2}}{\alpha_{0}}\right)-\frac{2}{3} I_{2,1}
$$

The stable single-mode operation can be reached if the loss for mode 2 is greater than the gain, i.e. mode 2 energy buildup is negative. In this case $d E_{2} / d t<0$, and the condition for the stable single-mode operation that loss for mode 2 must be as follows: $\gamma_{2}>\frac{1}{3} \alpha_{0}+\frac{2}{3} \gamma_{1}$.

It is clear from this condition that the main mechanism for the transition from single to multimode is spartial hole burning.

\section{Tuning the wavelength of the fiber laser}

Continuous tuning of the single mode fiber laser, which utilizes intracore Bragg reflectors for cavity feedback, can be achieved when both the gratings and intervening fiber are stretched and / or heated uniformly [8]. In this case, longitudinal mode hopping is eliminated since the change of the reflected Bragg wavelength tracks the change of the cavity resonance wavelength. In general, the fiber laser resonator of length $L_{c}$ will lase at wavelengths:

$$
\lambda_{0}=\frac{2}{m} n L_{c}
$$

where $\lambda_{0}$ is a resonance wavelength, $\mathrm{n}$ is the refractive index, and $\mathrm{m}$ is an integer. A change in the optical path length of the fiber, due to either heating or stretching the fiber, will result in a change of the resonant wavelength given by

$$
\frac{\delta \lambda_{0}}{\lambda_{0}}=\frac{\delta n}{n}+\frac{\delta L_{c}}{L_{c}}=\left(1-\rho_{e}\right) \varepsilon+(\eta+\zeta) \Delta T
$$

where $\eta$ is the coefficient of linear expansion, $\zeta$ is the thermo-optic coefficient, $\rho_{e}$ is the photoelastic constant, and $\varepsilon$ is the applied strain.

The effects of strain or thermal variation applied to a intracore Bragg reflector may be derived from the Bragg condition, which relates the wavelength of the reflected radiation at line center $\lambda_{B}$ to the grating period $\Lambda$. The Bragg condition is given by (3) and the change in Bragg wavelength as a function of strain or thermal variation applied to the fiber grating is given by

$$
\frac{\delta \lambda_{B}}{\lambda_{B}}=\frac{\delta n}{n}+\frac{\delta \Lambda}{\Lambda}=\left(1-\rho_{e}\right) \varepsilon+(\eta+\zeta) \Delta T
$$


As seen from (13) and (14), when the strain and/or thermal variation applied to both the Bragg reflectors and the intervening fiber is equal, the change in Bragg wavelength will match the change in cavity resonance wavelength. Since the single-mode fiber laser only lases in the lowest-loss mode, the wavelength of the lowest-loss mode will track the change in cavity resonance and tune smoothly without mode hopping. This assumes that the gain is relatively flat over the bandwidth of the grating, typically smaller than $0.2 \mathrm{~nm}$, which is the case in our Erbium-doped fiber laser.

\section{EXPERIMENT AND DISCUSSION}

An experimental DBR Fiber Laser scheme is illustrated in Fig. 2. The 1.55- $\mu \mathrm{m}$ DBR erbium doped fiber laser was fabricated in hydrogenated erbium-doped germanosilicate fiber. The sensitivity of a fiber increased 100 times after keeping under pressure in hydrogen gas. The fiber is highly doped with erbium concentration of approximately 1400 ppm. A laser cavity was formed between two Bragg grating reflectors, written directly in the fiber core, where the cladding was primarily took off. The gratings had been written non invasively using the holographic phase mask technique. The phase mask has a period of $1066.1 \mathrm{~nm}$. The gratings were written approximately $1 \mathrm{~cm}$ apart, by exposing through the mask to the second harmonic of an argon laser operating at a wavelength of $244 \mathrm{~nm}$. A free spectral range of this 1 -cm cavity is about $0.08 \mathrm{~nm}$, which satisfies the single mode condition of the laser. The Bragg reflectivity was achieved under exposure of an average $\mathrm{UV}$ power of $30 \mathrm{~mW}$ and a temperature of $25^{\circ} \mathrm{C}$. Both gratings have similar periods to ensure optimum wavelength overlap. One Bragg grating of $7.5 \mathrm{~mm}$ length is saturated. The other $5.5 \mathrm{~mm}$ is fine with reflectivity of about 90 percent to get single mode operation. Their principal behaviors are presented in Fig. 3.

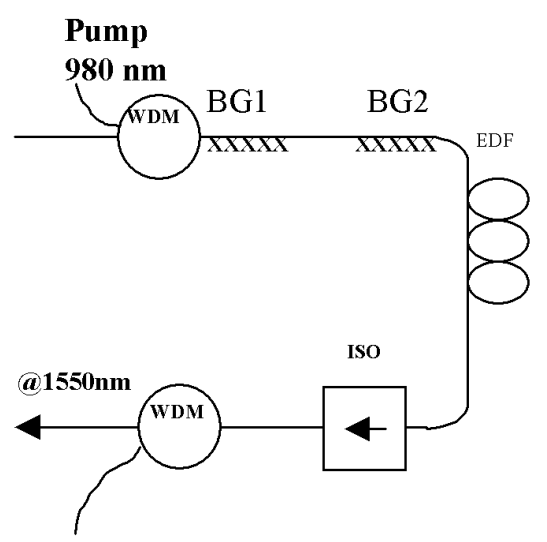

Fig. 2. Experimental setup of the DBR fiber laser with EDFA stage

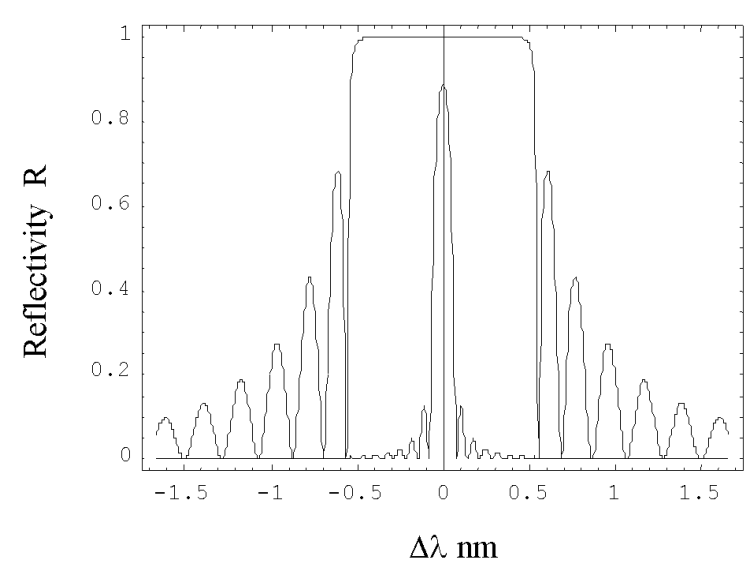

Fig. 3. The principal behaviours of two Bragg gratings reflectors.

The fiber laser was pumped at $980 \mathrm{~nm}$ with a SDL Semiconductor Laser Diode Model SDLO-1564-120 to give an DBR output power of $40 \mu \mathrm{W}$. The dependence of the DBR laser output power on the pump power is represented in Fig. 4. The fiber laser threshold 
was rather high $\approx 15 \mathrm{~mW}$, even for the very short cavity length of doped fiber, related to the losses due to high concentration of erbium ions and absorption by two extensions of fibers beyond the laser cavity. High concentration of doped erbium ions increases the effect of energy transferring between neighboring ions, which leads to non-radiatively loss of excited ions. The slope efficiency of the fiber laser, defined as the slope of the laser output power as a function of the injected pump power, was measured to be approximately $0.05 \%$. The low slope efficiency accounts for the fact that most of the pump power is not absorbed by the fiber but is transmitted through the fiber. This suggests that an ideal application for this fiber laser would be better with next stage of an EDF amplifier. The amplifier stage gives the gain of about $30 \mathrm{~dB}$, without any adding external pump.
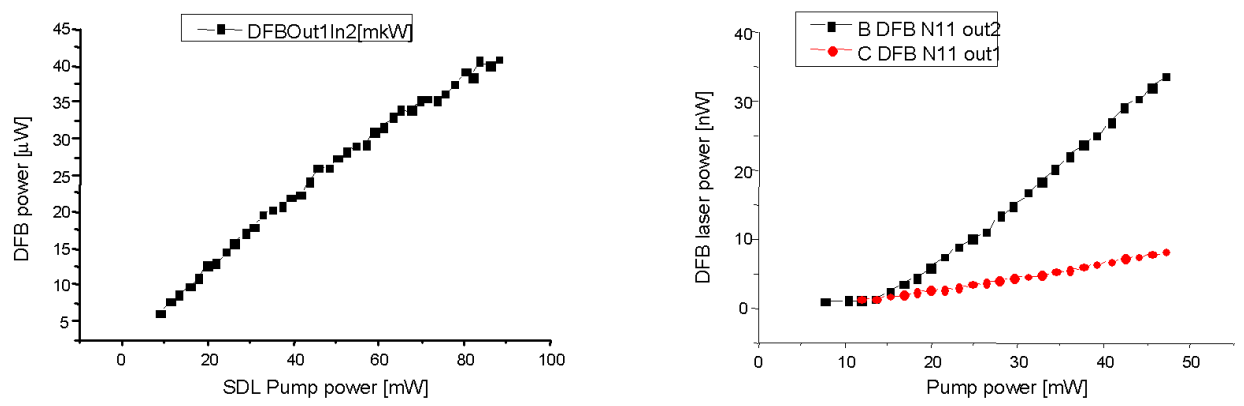

Fig. 4. The output power characteristic of the DBR fiber laser vs the pump power; Right: Initial characteristic of the DBR laser from two outputs for determining a threshold.

The spectrum of DBR fiber laser was measured by a spectrometer TRIAX320 with resolution $0.18 \mathrm{~nm}$ in IR range. Fig. 5 represents a spectrum of the DBR Laser at the pump power of $45 \mathrm{~mW}$. The full width at half maximum of the peak is about $0.2 \mathrm{~nm}$, which is limited by the instrument resolution. The measurement by scanning FabryPerot etalon $10 \mathrm{GHz}$ (NewPort) gave fine structure of the spectrum in Fig. 6.

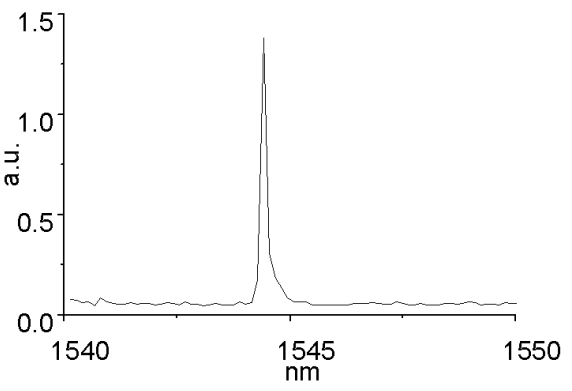

Fig. 5. Spectrum of the DBR laser

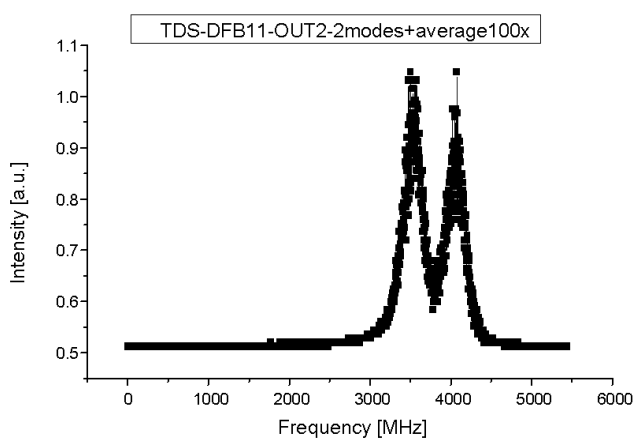

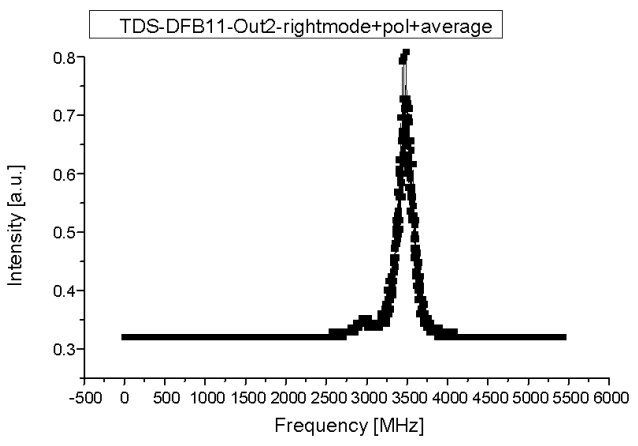

Fig. 6. Left - The spectrum of DBR laser measured by $10 \mathrm{GHz}$ Fabry-Perot, with two perpedicularly linear polarized peaks. Right - right peak separated by a polarizator 
There are two peaks related to two mutual perpendicular polarizations. This induced anisotropy appeared when the fiber was exposed by UV light. With a coil of Lefevre these peaks could be selected (right picture). The distance between these two modes is about $403 \mathrm{MHz}\left(\approx 3.22 \times 10^{-3} \mathrm{~nm}\right)$, and the FWHM of the line is about $150-200 \mathrm{KHz}(\approx 1.22$ $\left.4 \times 10^{-6} \mathrm{~nm}\right)$.

The laser signal is not continuous, even when the pump is continuous. This phenomenon belongs to heavily concentration of $\mathrm{Er}^{3+}$-ions in fiber used in DBR laser. It leads to the establishment of $\mathrm{Er}^{3+}$-ion clusters, and the transfer energy between two neighboring ions occurs $[9,10,11]$. The DBR laser became self-pulsing and presented in Fig. 7.

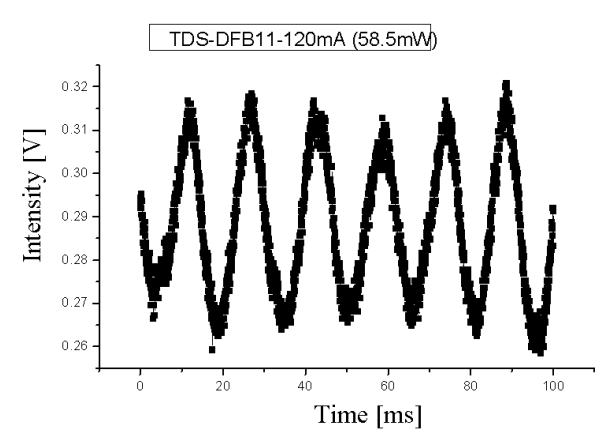

Fig. 7. Selfpulsing in DBR laser

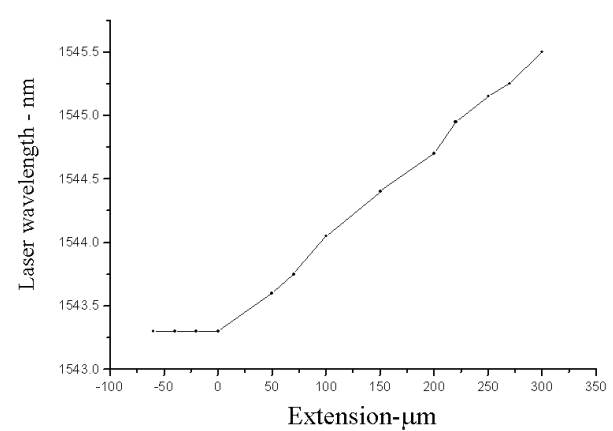

Fig. 8. Spectral tuning versus the extension of the DFB laser

The tuning operation was carried out by changing the strain. The strainer is a micrometer fixed firmly to one of the fiber mounts. The distance between two fiber mounts is $22 \mathrm{~cm}$. Continuous tuning was verified with the spectrometer TRIAX320. When stretch the micrometer by $500 \mu \mathrm{m}$, an wavelength shift of $\Delta \lambda=2.108 \mathrm{~nm}(\Delta \nu=263.2 \mathrm{GHz})$ was realized. This is consistent with the fact that the spacing between the mounting points was approximately $22 \mathrm{~cm}$ and led to $\delta \lambda / \lambda \approx 0.82 \varepsilon$, as shown in Fig.8. For fused silica, the effective photoelastic constant is 0.22 , so $\delta \lambda / \lambda$ is calculated to be $0.78 \varepsilon$. Core doping, however, can change the photoelastic constant.

In this experiment, the tuning of the fiber laser was limited by the proof test strength of the fiber.

\section{CONCLUSION}

A continuously tunable, single - mode DBR erbium fiber laser, which utilizes intracore Bragg reflectors for cavity feedback, has been demonstrated. The output power is reached $40 \mu \mathrm{W}$, a slope efficiency is $0.05 \%$, the redundant pump power may be used for pumping next EDF amplifier stage. The gain is reached $30 \mathrm{~dB}$ without any extra pump. The signal included two perpendicularly polarized modes, because of induced anisotropy brought by exposure of UV light. The laser is self - pulsing, even when the pump is continuous, due to high $\mathrm{Er}^{3+}$ concentration. The confirmed range of continuous tuning was $2.108 \mathrm{~nm}$. The full continuous tuning range must be maintained more with whole possibility strain corresponding to $1000 \mu \mathrm{m}$ extension. 


\section{ACKNOWLEDGEMENT}

The Natural Science Council of Vietnam and Research Program of the NCST supported this work. The authors wish to acknowledge Professor Nguyen Van Hieu - NCST for his valuable support.

\section{REFERENCES}

1. M. Digonnet, ed., Rare Earth Doped Fiber Lasers and Amplifiers, Marcel Dekker, New York, 1993

2. A. Gloag et al., J. Opt. Soc. Am. B, 13 (5) (1996) 921-925.

3. P. Barnsley, J. Opt. Soc. Am. A, 5 (1988) 1339-1346.

4. S. L. Gilbert, Opp. Let., 16 ( 1991) 150-152.

5. G. A. Ball and W. H. Glenn, J. of Lightwave Tech., 10 (1992) 1338-1343.

6. T. Erdogan, J. of Lightwave Tech., 15 (8) (1997) 1277-1294.

7. B. E. A. Saleh, M. C. Teich, Fundamentals of Photonics, John Wiley \& sons, Inc., New York,

8. Oliver Hadeler, Erland Ronekleiv, Morten Ibsen, and Richard I. Laming, Appl. Opt. 38 (10) (1999) 1953-1958.

9. P. Le Boudec, M. Le Flohic, P. L. Francois, F. Sanchez, G. Stephan, Optical and Quantum Electronics, 25 (1993) 359-367.

10. P. Le Boudec, P.L. Francois, E. Delevaque, J. F. Bayon, F. Sanchez, G. Stephan, Optical and Quantum Electronics, 25 (1993) 501-507.

11. S. Colin, E. Contesse, P. Le Boudec, G. Stephan, and F. Sanchez, Optics Letters, 21(24) (1996) 1987-1989.

Received 24 July 2003 